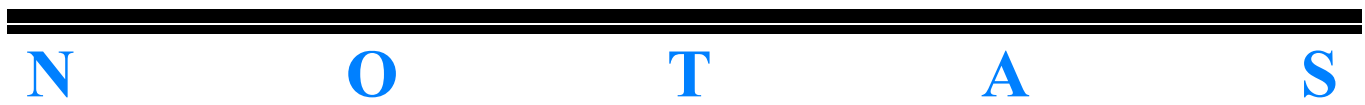

S 


\section{RONALD DWORKIN Y LAS \\ CONSECUENCIAS DE LA VAGUEDAD}

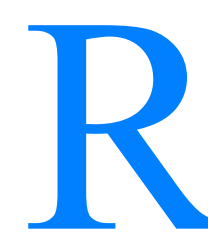

onald Dworkin sostiene que siempre hay virtualmente una única respuesta correcta para toda disputa jurídica ${ }^{1}$. Una posible objeción a su tesis es que si el lenguaje de una norma jurídica es vago, entonces no hay respuesta a la pregunta de si la norma se aplica a un caso marginal. Dworkin responde que la objeción no consigue interpretación eliminarían la vaguedad, por ejemplo por requerir que la norma sea aplicada sólo a casos «en el núcleo de certeza del lenguaje» ([4], pp. 67-9).

Joseph Razha replicado que Dworkin caracteriza erróneamente los términos vagos como si pudieran trazarse dos líneas precisas: una entre los casos claros y los casos dudosos, y otra entre los casos dudosos y los casos a los que el término es indiscutiblemente inaplicable. Raz argumentaba que normalmente no hay líneas entre casos claros y casos que no lo son y que, en el tipo central de vaguedad, «la vaguedad es “continua"» ([7], pp. 73-4). Recientemente, Brian Bix ha señalado la misma objeción, invocando el argumento de Mark Sainsbury según el cual la mejor forma de caracterizar la vaguedad es como careciendo de límites ([1], pp. 31-2; ver Sainsbury [8]). En la terminología de los análisis filosóficos recientes de la vaguedad, el argumento de Dworkin ignora el problema de la vaguedad de orden superior.

La objeción es válida. Pero no se refiere expresamente a una versión elaborada del argumento que Dworkin desarrolló para defender su pretensión ([2]). Argumentaré que la versión elaborada no consigue su propósito. Muestra, sin embargo, que alguien que pretenda que no hay respuesta correcta en un caso marginal de aplicación de una palabra vaga no debe formular tal pretensión diciendo que una aplicación de la palabra en tal caso no es «ni verdadera ni falsa».

\footnotetext{
${ }^{1}$ Véase [5] para una defensa reciente de la tesis.
} 


\section{La versión elaborada del argumento de Dworkin}

En la notación de Dworkin, $\underline{V}$ dice que, si `es vago, «x es `» puede ser verdadero, falso o ni verdadero ni falso. $\underline{V}$ pretende que, en la última de las tres opciones, el uso de `en la formulación de una norma convierte al derecho en indeterminado. Dworkin dice que el resultado no es necesariamente la indeterminación en el derecho: por ejemplo, un «principio de legislación» podría requerir que «x es '» sea tratado como falso si no es verdadero ([2], p. 139). Cuando «x es " no es ni verdadero ni falso, entonces no es verdadero. Por lo tanto, la regla de interpretación (que llamaré «regla de Dworkin») parece eliminar la indeterminación alegada por $\underline{\mathrm{V}}$.

Ahora, el nuevo oponente de Dworkin, $\underline{\mathrm{R}}$, pretende que no hay límites precisos entre casos claros y casos marginales, esto es, en los términos de $\underline{\mathrm{V}}$, “"x es '” es verdadero» puede que no sea ni verdadero ni falso. Pero Dworkin rechaza esta pretensión: si " $\mathrm{x}$ es ' " no es ni verdadero ni falso, entonces «"x es '” es verdadero» es falso. De nuevo entra en acción la regla de Dworkin, no hay ningún caso en el cual «"x es '” es verdadero» no sea ni verdadero ni falso.

$E$ El argumento deja la impresión de que $\underline{\mathrm{V}}$ ha puesto a $\underline{\mathrm{R}}$ en dificultades. Dworkin admite que la situación de $\underline{\mathrm{R}}$ es delicada, pero concluye que $\underline{\mathrm{R}}$ está comprometido con el punto de vista de $\underline{\mathrm{V}}$ : 130).

De esta forma $\underline{\mathrm{R}}$ parece ser víctima de la formulación misma del argumento de $\underline{\mathrm{V}}$. ([2], $\mathrm{p}$.

$\mathrm{Al}$ lector le puede parecer que $\underline{\mathrm{R}}$ ha sido engañado por este argumento. En cualquier caso, las circunstancias sobre las que $\mathrm{R}$ llamó la atención bien pudieran haber surgido a través de esta compleja argumentación. Alguien ha dicho que si no es verdad que un contrato es sacrílego tiene que tratarlo como no sacrílego, pero aún así puede encontrarse con el problema de no estar seguro del hecho de que no sea verdad que el contrato en cuestión es sacrílego. Estoy de acuerdo. Pero este es un problema para $\underline{\mathrm{V}}$, no para mi respuesta a $\underline{\mathrm{R}}$. ([2] n. 405 n. 3).

El problema de $\underline{\mathrm{R}}$ es el presupuesto de $\underline{\mathrm{V}}$ (que Dworkin señala en [2], p. 130) según el cual cuando es indeterminado si «x es `» es verdadero, «x es `» no es verdadero. El esquema trivalente de $\underline{\mathrm{V}}$ (en el que «x es “» puede ser verdadero, o falso, o ni verdadero ni falso) presupone bivalencia para «"x es '” es verdadero» (i.e., cualquier emisión del último enunciado debe ser o bien verdadera o bien falsa $)^{2}$. La caracterización trivalente de la vaguedad frustra al desventurado $\underline{\mathrm{V}}$ y al perplejo $\underline{\mathrm{R}}$.

${ }^{2} \mathrm{R}$ no puede salir de este atolladero ni siquiera postulando un número infinito de valores de verdad, correspondientes podemos decir a los números reales entre 0 y 1 . Un principio de interpretación similar a la regla de Dworkin podría eliminar la vaguedad, requiriendo que la regla sea aplicada sólo cuando el grado de verdad del antecedente de la regla fuere 1 . 


\section{2. ¿Cuál es el error de V?}

El error de V está en decir que, en un caso marginal, «x es `» no es ni verdadero ni falso. El error de Dworkin es asumir que la pretensión de que la vaguedad puede llevar a la indeterminación en un caso particular debe ser discutida en aquellos términos: que aquél que no sostiene la bivalencia debe sostener la trivalencia. Podemos hallar una forma mejor de formular la pretensión si extraemos el núcleo de buen sentido del argumento de $\underline{\mathrm{V}}$.

La caracterización de $\underline{\mathrm{V}}$ es puramente negativa. Podemos reformularla de dos formas, cada una de ellas otorga un alcance diferente a la negación de $\underline{V}$. La primera presenta la negación de $\underline{V}$ como externa a la aserción de que $\mathrm{x}$ es ' $\mathrm{y}$ la segunda la presenta como interna a dicha aserción:

1) negación externa: al decir «" $x$ es '” no es ni verdadero ni falso», $\underline{V}$ rechaza la afirmación de que «x es `» es verdadero o falso;

2) negación interna: al decir «"x es '” no es ni verdadero ni falso», $\underline{V}$ afirma a la vez que «x es "» no es verdadero y que no es falso.

La primera opción otorga sentido a la pretensión de $\underline{\mathrm{V}}$ según la cual, en un caso dudoso, «X es '» no es ni verdadero ni falso. Un caso dudoso es aquel en el cual un hablante competente e informado no sabría si afirmar que $\mathrm{x}$ es '.

Si consideramos la pretensión de $\underline{\mathrm{V}}$ de esta forma, la regla de Dworkin no elimina la vaguedad. Reformulado en términos de la negación externa, la regla de Dworkin es que «x es ¿ debe ser tratado como falso cuando los hablantes competentes e informados no estarían dispuestos a afirmar que es verdadero. La susceptibilidad de ser aseverado es vaga: podría haber casos dudosos de la aplicación de una norma según la cual los contratos son inválidos si no puede ser aseverado que son sacrílegos. Por lo tanto, si tratamos la pretensión de $\underline{\mathrm{V}}$ como una negación externa, $\underline{\mathrm{R}}$ consigue mostrar que la regla de Dworkin reduce la indeterminación, pero no la elimina.

La negación externa no es una operación lógica, sino una figura retórica. La construcción de la pretensión de $\underline{\mathrm{V}}$ como una negación externa conduce a una forma más bien engañosa de decir que no es claro si x es '. Es engañosa porque es fácil confundirla con la negación interna.

La negación interna es la negación familiar en la lógica tradicional. La lógica tolera la negación externa de pretensiones de conocimiento y de otras operaciones modales, tales como «No sé si ella está en casa» que difiere de «Sé que ella no está en casa» de la misma forma en la lógica modal que en el discurso ordinario. De forma semejante, la lógica distingue entre la negación externa y la interna de las expresiones cuantificadas, esto es «es el caso que todos los grifos no son monógamos» difiere de «no es el caso que todos los grifos son monógamos» de la forma familiar en lógica. Pero la lógica clásica no tiene lugar para la negación externa de aserciones. La única negación de la aserción $\mid \mathrm{p}$ es $\mid \neg \mathrm{p}$; no hay fórmula $\mid \neg \mathrm{p}$ : el signo de 
aserción pertenece al metalenguaje y el signo de negación pertenece al lenguaje objeto.

Dworkin piensa en la negación interna en su argumento contra $\underline{\mathrm{V}}$. Define la «segunda versión de la tesis de que no siempre hay respuesta correcta» (en favor de la cual argumenta $\underline{\mathrm{V}}$ ) en términos de «negación lógica», explicando que $\neg p$ ) es la negación lógica de (p), «de forma que si (p) es falso ( $\neg$ p ) es verdadero, y si $(\neg p)$ es falso (p) es verdadero» ([2], p. 12). Y señala que la segunda versión de la tesis de que no siempre hay respuesta correcta «sostiene que en algunos casos ni (p) ni $(\neg \mathrm{p})$ son verdaderos, esto es, que en algunos casos la bivalencia no se mantiene» ([2], p. 122).

Pero si el argumento de $\underline{\mathrm{V}}$ usa la negación interna, $\underline{\mathrm{V}}$ tiene un problema peor que el de la regla de Dworkin. $\underline{\mathrm{V}}$ pretende que en algunos casos, «x es '» no es verdadero y que no es falso. Si aceptamos la definición de Dworkin de negación lógica y el principio trivial según el cual si «x es `» es verdadero, entonces $\mathrm{x}$ es ` ( $\mathrm{y}$ V) que si «x es `» es falso, entonces x no es ` (y V), entonces la pretensión de $\underline{\mathrm{V}}$ según la cual «x es " no es ni verdadero ni falso se contradice a sí misma.

Timothy Williamson ha demostrado la contradicción en un argumento por reducción al absurdo a que conduce la denegación de la bivalencia, puesto que ello conlleva negar la ley del tercero excluido:

(T) Si $\underline{u}$ dice que $\underline{p}$, entonces $\underline{u}$ es verdadero si y sólo si $\underline{p}$ [esquema desentrecomillado para la verdad $\left.{ }^{3}\right]$.

(F) Si $\underline{u}$ dice que $\underline{p}$, entonces $\underline{u}$ es falso si y sólo si no $\underline{p}$. [ídem para la

(O) $\underline{u}$ dice que $p$. [definición]. falsedad].

(1) No: o bien $\underline{u}$ es verdadero o bien $\underline{u}$ es falso [negación de la bivalencia; cf. la pretensión de $\underline{\mathrm{V}}$.

(2a) $\underline{u}$ es verdadero sí y sólo si $\underline{p}$.

$$
[(\mathrm{T}),(1)] \text {. }
$$

(2b) $\underline{u}$ es falso si y sólo si no $\underline{p}$.

$$
[(\mathrm{F}),(1)] \text {. }
$$

(3) No: o bien p o no p. [sustitución de (2a) y (2b) en (1)].

(4) No $p$ y no no p.. ${ }^{4}$ [(3), leyes de Morgan].

Entonces la pretensión de $\underline{\mathrm{V}}$ según la cual «x es `» no es ni verdadero ni falso implica la pretensión incoherente según la cual ni `ni no ’, lo que es lo mismo, a la vez `y no `.

Hay una segunda manera, más simple, de exponer la incoherencia en la pretensión de $\underline{\mathrm{V}}$ cuando es caracterizada como una negación interna: si no es claro si x es `, no es claro que «x es '» no es verdadero y no es claro que

\footnotetext{
${ }^{3}$ Para el cual Williamson apela a Tarski y a Aristóteles: [10] p. 188.

${ }^{4}[10]$, pp. 188-189; yo he añadido las glosas.
} 
no es falso. No sólo los dos conjuntos de la pretensión de $\underline{\mathrm{V}}$ son lógicamente inconsistentes, sino que $\underline{\mathrm{V}}$ ni siquiera puede afirmar que uno de ellos sea el caso. ${ }^{5}$

Entendida en términos de negación interna, entonces, la pretensión de $\underline{\mathrm{V}}$ tiene la significación retórica de una contradicción emitida con una función de énfasis, pero no desempeña ningún papel en el análisis lógico. Construir la pretensión de $\underline{\mathrm{V}}$ como una negación interna y considerarla como una contradicción usada retóricamente tiene el mismo efecto que construirla como una negación externa: la pretensión de $\underline{\mathrm{V}}$ tiene significado sólo en sentido figurativo. Tal vez la moraleja es que $\underline{\mathrm{V}}$ no debería formular la pretensión de indeterminación diciendo que un enunciado no es verdadero ni falso en un caso marginal, sino diciendo que no es claro si el enunciado es verdadero.

¿Cuál es la conclusión para Dworkin?

Dado que es contradictoria, la pretensión de $\underline{\mathrm{V}}$ como una negación interna no proporciona a la regla de Dworkin nada en que continuar trabajando. Formulada como una negación externa, la pretensión de V no es afectada por la regla de Dworkin, por la razón que él admite: puede no ser claro si, e.g. un contrato es un caso marginal de la aplicación de «sacrílego», así como puede no ser claro si un contrato es sacrílego. Este es un problema para Dworkin (y no sólo un problema para $\underline{\mathrm{V}}$ ), porque muestra que la regla de Dworkin no puede eliminar esa falta de claridad.

Podría objetarse que Dworkin tiene razón al pensar que $\underline{\mathrm{R}}$ está comprometido con la formulación de $\underline{\mathrm{V}}$ si $\underline{\mathrm{R}}$ afirma que no hay respuesta correcta: $\underline{\mathrm{V}}$ afirmaba que no hay respuesta correcta cuando decía que «x es `» no es ni verdadero ni falso. Argüir que $\underline{V}$ no debería decir que «x es `» no es ni verdadero ni falso parece equivalente a argüir que no debería decir que no hay respuesta correcta. Sin embargo, si es indeterminado si x es ', entonces no hay respuesta correcta a la pregunta si $x$ es '. No es necesario sostener la trivalencia para negar que hay una respuesta correcta, no más que para

${ }^{5}$ Es necesario destacar que pueden construirse lógicas trivalentes en las que no hay contradicción en decir que un enunciado no es verdadero ni falso: para un análisis de estas lógicas que se remontan a C. S. Peirce, véase Williamson [10], capítulo 4. Un ejemplo ulterior es la lógica de la verdad de G. H. van Wright ([9]; estoy agradecido a José Juan Mores y Pablo Navarro por llamarme la atención sobre este trabajo). Tales lógicas presentan una tercera opción: presentar la pretensión de $\mathrm{V}$ como una negación externa usando una lógica no-clásica que admite negaciones externas (la lógica de la verdad, por ejemplo, representa la verdad mediante un operador modal que permite distinguir en su lógica entre la negación interna y la externa de aserciones). Algunas posibles objeciones al uso de lógicas trivalentes para el tratamiento son las siguientes: (i) estas lógicas sustituyen la noción ordinaria de «verdadero» por algo cercano a la noción ordinaria de «claramente verdadero», (ii) no pueden dar cuenta de la vaguedad de orden superior, (iii) deben rechazar o bien la ley del tercero excluido o los plausibles (T) y (F) de Wiliamson y (iv) ignoran la razón dada justamente para no afirmar que la emisión de un enunciado en un caso marginal no es ni verdadera ni falso. Estas objeciones no pueden ser desarrolladas aquí, es suficiente para el argumento presente el hecho de si $\mathrm{R}$ tiene alguna alternativa disponible a la lógica trivalente que $\mathrm{V}$ parece trazar. 
pretender que no hay un último segundo del crepúsculo de una tarde deba decirse que algo se interfiere entre el crepúsculo y la oscuridad.

\section{Vaguedad como ignorancia}

El problema teórico con la vaguedad es como caracterizar la falta de claridad en un caso marginal. El punto de vista más simple es que podríamos caracterizarla como «indeterminación» y decir que, en un caso marginal, el significado de la palabra no determina si ésta se aplica o no. Un punto de vista alternativo es que la falta de claridad no es una cuestión de significado, sino de ignorancia, que el significado de una palabra puede determinar las condiciones de aplicación sin conocimiento de los individuos, y que en la aplicación de palabras vagas a casos marginales, los individuos simplemente no saben si la palabra se aplica. Desde este punto de vista epistémico de la vaguedad, habrá una única respuesta correcta aún en los casos en que es incierta la aplicación del lenguaje vago de la formulación de la norma ${ }^{6}$.

Desde este punto de vista epistémico, toda cuestión acerca de la aplicación de una regla vaga tiene una respuesta correcta. Un contrato es sacrílego, o bien, no es sacrílego, con los límites establecidos por el significado de la palabra «sacrílego». El problema en casos marginales no es que no existan límites, sino que no sabemos en dónde están. Hay una respuesta correcta, pero los jueces no pueden contar con encontrarla en todos los casos.

Mediante la adición de una teoría acerca de cuando (si alguna vez) un juez debe apartarse de la extensión precisa de las palabras en las cuales el derecho es formulado, el punto de vista epistémico podría convertirse en una teoría de la aplicación judicial de las normas formuladas en un lenguaje. Pero tal teoría sería repugnante para la visión del derecho de Dworkin. En primer lugar, esta teoría epistémica de la decisión judicial es una teoría semántica: considera la aplicación del derecho como determinada por el significado de las palabras en las cuales éste es formulado. Dworkin presenta su teoría interpretativa en oposición a tales teorías (véase [3], pp. 31-32, 45-46). En segundo lugar, para algunos conceptos el punto de vista epistémico de la vaguedad está reñido con una distinción que Dworkin establece entre conceptos y concepciones. Dworkin considera a los conceptos como vacíos de contenidos, aunque funcionando como plateaus en el cual compiten las concepciones ${ }^{7}$; según el punto de vista epistémico, la extensión de un concepto determina todas las cuestiones acerca de la aplicación de una palabra. En tercer lugar, el juez ideal de Dworkin, Hércules, no

${ }^{6}$ Para una presentación del punto de vista epistémico de la vaguedad, véase Williamson [10], especialmente capítulo 7.

${ }^{7}$ [3], pp. 70-2. Acerca de la distinción entre conceptos y concepciones, véase también Dworkin [6], pp. $134-6$, y [2], p.128. 
afronta un obstáculo epistémico para encontrar la respuesta correcta en todos los casos. No porque Hércules tenga un privilegio epistémico sobre los demás jueces; simplemente tiene más tiempo y un enfoque holístico de la adjudicación más consciente (véase, e.g., [2], pp. 264-5).

Finalmente, el punto de vista epistémico no pierde el tiempo con la trivalencia, y es solamente la trivalencia de $\underline{\mathrm{V}}$ lo que da lugar a la distinción de Dworkin entre la vaguedad del lenguaje de las normas y las consecuencias de ese lenguaje para el derecho. Según el punto de vista epistémico, al igual que en la indeterminación semántica, la regla de Dworkin no eliminará la vaguedad.

\section{Conclusión}

La característica más irónica de la paradoja del montón es que el alcance de lo determinado es indeterminado. Esto es, cualquier frase de la forma «A B» será vaga, donde $\mathrm{B}$ sea un adjetivo vago y A sea un adverbio mediante el cual tratemos de eliminar la vaguedad de $\mathrm{B}$ (tal como «determinadamente», o «claramente» o «definitivamente» $\mathrm{o}$ «indisputablemente» o «verdaderamente»). Un buen tratamiento de la vaguedad considerará tales frases como vagas. Dado que no es claro si algunos casos están en el núcleo de aplicación de una palabra, una regla de interpretación que requiera que una norma sea aplicada solamente en casos claros no puede eliminar la vaguedad. La consecuencia de la vaguedad en la formulación de las normas jurídicas es la indeterminación en el derecho ${ }^{8}$.

Esa conclusión no debe ser formulada en términos de que los enunciados no son verdaderos ni falsos en los casos marginales. Tal formulación puede tener sentido sólo como una manera engañosa de decir que es indeterminado si el enunciado es verdadero. Una pretensión de que la verdad de un enunciado es indeterminado en un caso marginal no debe ser entendida como introduciendo un nuevo valor de verdad, sino como afirmando que la incertidumbre acerca de si el enunciado es verdadero es una cuestión del significado del enunciado, y no sólo una cuestión de ignorancia.

Todavía resta destacar el alcance limitado por el cual este debate puede (1) favorecer o (2) dificultar la tesis de Dworkin de que hay virtualmente siempre una respuesta correcta a una cuestión jurídica.

1. Si la regla de Dworkin eliminase la vaguedad, el argumento de Dworkin solamente establecería que la indeterminación jurídica no resulta de la vaguedad en la formulación de una norma jurídica si tal regla de interpretación

${ }^{8}$ Sin embargo, dicha consecuencia no se produce si los tribunales reemplazan un estándar vago por uno preciso, e.g. mediante la especificación de un período temporal en el cual una acción sería considerada como si hubiese ocurrido «en» o «dentro de un tiempo razonable» conforme a los propósitos de una ley que usa tal expresión. 
es empleado. En realidad, los cánones de interpretación son expresados en un lenguaje vago, y tal vez no exista tal cosa como un canon preciso de interpretación. Dworkin responde que «aún dejamos abierta la cuestión de cómo el derecho es afectado por el hecho de que los jueces, en esos enunciados canónicos, han usado términos vagos $»{ }^{9}$, Pero el argumento de Dworkin de que la vaguedad no resulta en indeterminación jurídica apela a principios para la eliminación de esta vaguedad. Así, la vaguedad en formulaciones canónicas de cánones de construcción está fuera del alcance de ese argumento, a menos que existan reglas para la aplicación de los cánones de interpretación que eliminen su vaguedad. Y aquellas reglas de tercer orden deberían estar libres de vaguedad, o bien debería haber reglas para eliminar su vaguedad... Dworkin es confinado a enunciar que la vaguedad de una norma (de primer-orden) y la vaguedad de las reglas de interpretación «no significan que nuestra cuestión no tiene respuesta correcta... Nada ha sido todavía dicho, dependiente de la vaguedad del termino «sacrílego», para hacernos dudar acerca de si nuestra cuestión tiene respuesta correcta» ${ }^{10}$. Si tuviese éxito la versión elaborada de su argumento, Dworkin podría solamente sostener una pretensión que bien puede hacer sin ese argumento: la carga de la persuasión debe recaer sobre aquellos que pretenden que no hay una única respuesta correcta a una cuestión jurídica.

2. La tesis de Dworkin de la respuesta correcta es parte de su teoría del derecho como una interpretación constructiva de la práctica jurídica de una comunidad y no descansa en su argumento de que la vaguedad es eliminable mediante principios de interpretación. En realidad, Dworkin no considera muchas de las palabras aparentemente vagas empleadas en la formulación de estándares jurídicos («cruel», «justo»,...) como vagas en absoluto, sino que las considera como invocando conceptos que admiten diferentes concepciones. Nos previene acerca de confundir «el caso en el cual una legislatura usa un término vago, como «mediana edad»o «rojo», con el caso diferente en el cual se establece un concepto que admite diferentes concepciones $\gg{ }^{11}$. Tal vez Dworkin podría neutralizar la vaguedad al generalizar sus pretensiones acerca de tales conceptos (lo cual no puede ser considerado aquí) a todas las palabras empleadas en la formulación de estándares jurídicos. Parece, por ejemplo, que los individuos podrían tener diferentes concepciones acerca de lo que es mediana edad o rojo.

Pero esa táctica no erradicaría la vaguedad. Palabras tales como «cruel», «justo»y «cortesía», las cuales son ejemplos de Dworkin de conceptos que admiten diferentes concepciones, son vagas al menos en un importante sentido:

${ }^{9}[2]$, p. 130.

${ }^{10}[2]$, p. 131.

11 [2], p. 128. En Law's Empire ([3]), Dworkin parece mantener la misma distinción: considera a la vaguedad un «defecto semántico» (p. 17) y no ve ningún defecto semántico en palabras para conceptos que admiten diferentes concepciones. 
ellas comparten con palabras tales como «mediana edad» y «rojo», la característica de ser susceptibles de la aplicación de la paradoja de sorites, que conduce a la pretensión de que hay indeterminación en la aplicación de palabras vagas ${ }^{12}$.

(Trad. de José Juan Moreso y Pablo E. Navarro)

\section{REFERENCIAS}

[1] Brian Bix, Law, Language and Legal Determinacy (Oxford: Clarendon Press, 1993).

[2] Ronald Dworkin, «Is There Really No Right Answer in Hard Casos?», en Dworkin, A Matter of Principle (Oxford: Clarendon Press, 1986), pp. 119-145. i

[3] Ronald Dworkin, Law's Empire (Cambridge: Harvard University Press, 1986).

[4] Ronald Dworkin, «No Right Answer?» en Law, Morality and Society (Oxford: Clarendon Press, 1977), editado por P.M.S. Hacker y Joseph Raz, pp. 58-84.

[5] Ronald Dworkin, «On Gaps in the Law», in Controversies about Law's Ontology (Edinburgh: Edinburgh University Press, 1991), editado por Paul Amselek y Neil MacCormick, 84-90.

[6] Ronald Dworkin, Taking Rights Seriously, (Londres: Gerald Duckworth, 1977)

[7] Joseph Raz, The Authority of Law: Essays on Law and Morality (Oxford: Clarendon Press, 1979)

[8] Mark Sainsbury, «Is There Higher-Order Vagueness?», (1991) Philosophical Ouaterly 41, pp. 167-182.

[9] Georg Henrik van Wright, «Truth and Logic» en Truth. Knowledge and Modalities (Oxford: Basil Blackwell, 1984), 26-41.

[10] Timothy Williamson, Vagueness (Londres: Routledge, 1994)

12 Recluir e incomunicar a un individuo por 24 horas es cruel. Así también 23 horas y 59 minutos de incomunicación. Por una serie estándar de sorites podemos obtener la conclusión de que un minuto de incomunicación por día (o ningún minuto) es cruel. Series similares de sorites pueden ser construidas para «cortesía», «juste», etc. 
